\title{
The accumulation of metabolic syndrome components is associated with higher risk of positive surgical margin among patients with localized prostate cancer after radical prostatectomy
}

This article was published in the following Dove Medical Press journal: OncoTargets and Therapy

\author{
Xiaonan Zhengl,* \\ Shi Qiu ${ }^{1,2, *}$ \\ Xinyang Liao' \\ Xin $\mathrm{Han}^{3}$ \\ Kun Jin' \\ Lu Yang' \\ Qiang Wei'
}

'Department of Urology, Institute of Urology, West China Hospital, Sichuan University, Chengdu, Sichuan, People's Republic of China; ${ }^{2}$ Center of Biomedical Big Data, West China Hospital, Sichuan University, Chengdu, Sichuan, People's Republic of China; ${ }^{3}$ West China Medical School, Sichuan University, Chengdu, Sichuan, People's Republic of China

*These authors contributed equally to this work
Correspondence: Qiang Wei; Lu Yang Department of Urology, West China Hospital, Institute of Urology, Sichuan University, No 37. Guoxue Alley, Chengdu 61004I, Sichuan, People's Republic of China Tel +86 I89 8060 I425; $+86 \mid 3541235213$

Fax +86 288542245 ।

Email weiqiang933@I26.com; wycleflue@163.com
Objective: To evaluate the association between metabolic syndrome (MetS) and the accumulation of its components with prostate cancer $(\mathrm{PCa})$.

Patients and methods: Patients undergoing radical prostatectomy were retrospectively included. Patients were grouped by low risk and intermediate-high risk according to International Society of Urological Pathology grade. Multivariable logistic regression and Cox hazard regression model were utilized to assess the association of MetS with overall survival, biochemical recurrence, upgrading, upstaging, and positive surgical margin (PSM) after prostatectomy. Besides, trend test was also performed to evaluate the impact of the accumulation of MetS components on postoperative pathological feature.

Results: A total of 1,083 patients were eventually enrolled. With a median follow-up of 40.45 months, 197 patients were diagnosed with MetS. No significant association between MetS and survival outcomes and pathological features was found. However, we did notice that the accumulation of the MetS components could lead to an elevated gradient of the PSM risk in the entire cohort (one component: $\mathrm{OR}=1.46$; two components: $\mathrm{OR}=1.89$; $\geq 3$ components: $\mathrm{OR}=2.07 ; P$ for trend=0.0194) and intermediate-high risk group (one component: $\mathrm{OR}=1.4$; two components: $\mathrm{OR}=1.85 ; \geq 3$ components: $\mathrm{OR}=2.05$; $P$ for trend=0.0127).

Conclusion: The accumulation of MetS components could lead to increasing risk of PSM on the entire PCa cohort and patients with intermediate-high risk PCa after prostatectomy, but not for the low-risk patients.

Keywords: prostate cancer, PCa, metabolic syndrome, MetS, International Society of Urological Pathology, ISUP, positive surgical margin, PSM

\section{Introduction}

Prostate cancer (PCa) is the most frequently diagnosed malignant tumor in male population worldwide. ${ }^{1}$ Although the 5-year survival rate of PCa is approaching $100 \%, \mathrm{PCa}$ is still the third leading cause of cancer-specific death in men despite its indolent course and indiscriminate early prostate-specific antigen screening. ${ }^{2-6}$ Metabolic syndrome (MetS), which is composed of any three of the following metabolic abnormalities: hyperglycemia, obesity, hypertension, hypertriglyceridemia, and reduced high-density lipoprotein, is commonly believed to be associated with elevated risk of cardiovascular disease. ${ }^{6}$ In addition, MetS has been reported to play a role in the increased incidence 
of cancers. ${ }^{7}$ Citing a claim by Hursting, ${ }^{8}{ }^{89}$ MetS is linked with the risk and progression of $\mathrm{PCa}$ at biochemical level. In regard to the epidemiologic evidence, controversial results have been yielded. ${ }^{10,11}$ However, prior research concerning low-risk PCa patients treated with radical prostatectomy (RP) and MetS is inadequate. Previously, in several low-risk PCa cohorts, obesity and body mass index (BMI) were believed to be risk factors of $\mathrm{PCa}$ progression. ${ }^{12,13}$ A recent study reported that MetS was connected with higher probability of upgrading and positive surgical margin (PSM) in low-risk PCa patients treated with RP. ${ }^{14}$

This study aims to evaluate the impact of MetS and the number of its components on a PCa cohort undergoing RP, which is subsequently classified by low-risk and intermediate-to-high-risk groups according to International Society of Urological Pathology (ISUP) grade.

\section{Patients and methods}

Patients undergoing RP in our institution without prior $\mathrm{PCa}$ treatment or metastasis were identified $(n=1,167)$. Fifty-nine patients were excluded due to the lack of follow-up, and 26 patients refused to be included in the study. Nine patients without complete records were also excluded. Ultimately, we included 1,083 patients in this study. Demographic and clinicopathological parameters were collected retrospectively. All of the patients were restricted to MetS and non-MetS in ISUP grade 1 group (low risk, Gleason Score $[\mathrm{GS}] \leq 6, \mathrm{n}=74$ ) and ISUP grade $2-5$ group (intermediate to high risk, GS $\geq 7$, $\mathrm{n}=1,009$ ). Upstaging was defined as from cT1c/T2a to pathological T3a/N1 or higher, while upgrading was confirmed when postoperative ISUP grade was 1 or more grade higher than preoperative ISUP grade. This study was approved by the ethics committee of West China Hospital and informed consent was obtained from the patients. The patient consent was written informed consent, and this study was conducted in accordance with the Declaration of Helsinki.

MetS was assessed before 1) RP according to BMI ( $\geq 28 \mathrm{~kg} \mathrm{~m}^{-2}$ );2) elevated serum triglycerides ( $\geq 150 \mathrm{mg} \mathrm{dL}^{-1}$ or $1.7 \mathrm{mmol} \mathrm{L}^{-1}$ ), or treatment for this abnormality; 3) reduced serum high-density lipoprotein cholesterol (HDL-C) ( $<40 \mathrm{mg} \mathrm{dL}^{-1}$ or $1.03 \mathrm{mmol} \mathrm{L}^{-1}$ ), or treatment for this abnormality; 4) elevated blood pressure ( $\geq 130 / 85 \mathrm{mmHg}$ ), or use of antihypertensive medications; 5) elevated fasting glucose ( $\geq 100 \mathrm{mg} \mathrm{dL}^{-1}$ or $5.6 \mathrm{mmol} \mathrm{L}^{-1}$ ), or use of medications for hyperglycemia, or physician-diagnosed type 2 diabetes mellitus. ${ }^{6}$ Note that we used BMI rather than waist circumstance, which is in contrast to the previous study. ${ }^{15}$ The evidence supporting us to use BMI and waist circumstance in this study was these two anthropometric measures work similarly regarding overall biological association, given the correlation is fairly strong between them (Pearson correlation: 0.88-0.94 in men). ${ }^{16}$ We defined BMI over $28 \mathrm{~kg} \mathrm{~m}^{-2}$ as obesity based on the statement of Working Group on Obesity in China. ${ }^{17}$

Chi-squared test or Fisher's test and Kruskal-Wallis ranksum test were used to compare categorical and continuous variables. The association between MetS and the number of its components with pathological parameters was evaluated using a test for linear trend with covariables adjusted, for which we entered the median value of each category of the number of MetS components as a continuous variable in the models. Then we performed competing risk analysis to compare the association of the number of MetS components with PSM. Overall survival (OS) and biochemical recurrence (BCR)-free survival were also assessed by Kaplan-Meier curve and Cox hazard regression model with covariables adjusted.

$P$-value $<0.05$ was considered to be statistically significant. Statistical analyses were conducted by Empowerstats (X\&Y Solutions Inc., Boston, MA, USA).

\section{Results}

Baseline characteristics of the patients included in our study are presented in Table 1. A total of 1,083 patients were included, 190 (17.5\%) of which were MetS patients while other $893(82.5 \%)$ were not. In the entire cohort, MetS patients had a higher amount of 1-3 and 7-9 positive cores than non-MetS patients $(31.75 \%$ vs $29.68 \%$ and $26.19 \%$ vs $14.93 \%, P=0.007)$. On the contrary, non-MetS patients had a higher frequency of 4-6 and 10-12 positive cores than MetS patients $(29.68 \%$ vs $25.4 \%$ and $25.7 \%$ vs $16.67 \%$, $P=0.007)$. Similar outcome was observed in ISUP grade $2-5$ group $(P=0.011)$ but not in ISUP grade 1 group. MetS patients were reported to have higher BMI than non-MetS patients in all three groups (entire cohort: 25.66 vs 23.47 , $P<0.001$; ISUP $1: 25.96$ vs $23.32, P=0.003$; ISUP $2-5: 25.64$ vs $23.47, P<0.001)$. No significant difference was reported with regard to other factors.

Table 2 displays the adjusted multivariate logistic regression result for MetS for pathological outcomes. Upgrading and upstaging were more common among MetS patients than non-MetS in all groups, except upgrading in ISUP 1 group showed decreased odds. Increased adjusted OR for PSM was observed in ISUP 1 group. However, none of them were statistically significant.

BCR-free survival rate of the entire cohort was investigated by Kaplan-Meier curves and is shown in Figure 1. For 


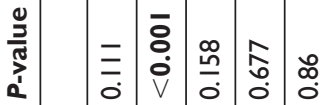

范

辛

我

壳

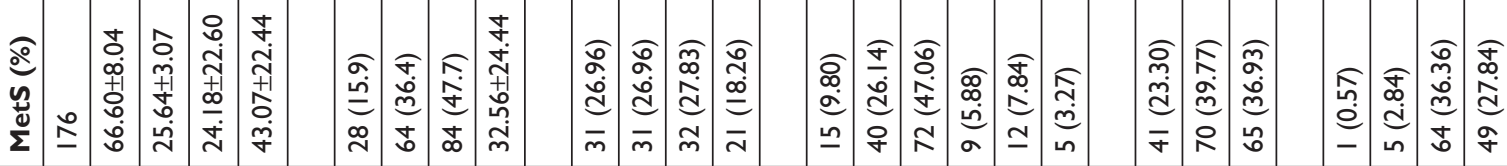

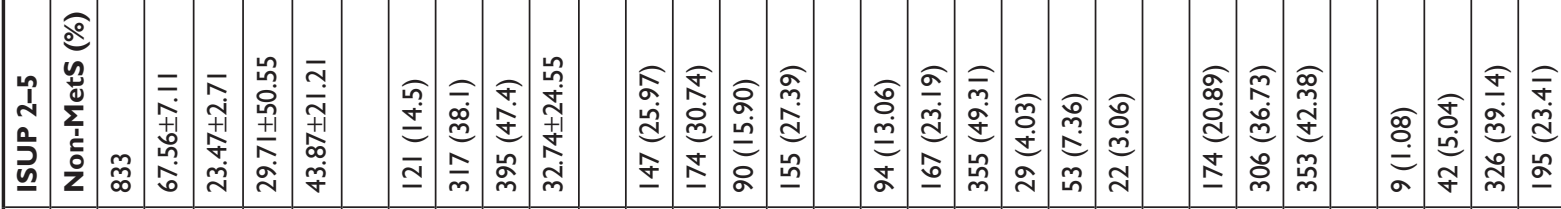

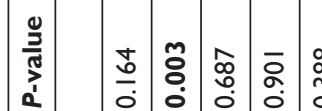

葛

$\stackrel{\infty}{\infty}$

$\underset{\substack{0 \\ \hdashline}}{\infty}$

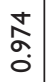

\begin{tabular}{|c|c|c|c|c|c|c|c|c|c|c|c|c|c|c|c|c|c|c|c|c|c|c|c|c|c|}
\hline ฮ̊ & & 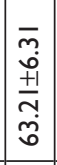 & & $\mid$ & & & 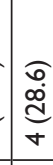 & 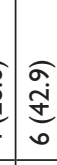 & $\begin{array}{l}\infty \\
\stackrel{\infty}{0} \\
\stackrel{p}{q} \\
\frac{1}{+}\end{array}$ & & 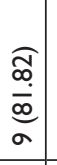 & 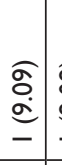 & 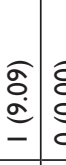 & & 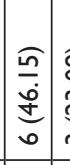 & 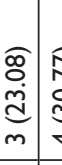 & & $\begin{array}{c}5 \\
5 \\
5 \\
\end{array}$ & $\begin{array}{l}0 \\
\dot{\Delta} \\
\dot{O} \\
\circ\end{array}$ & & $\begin{array}{l}0 \\
\delta \\
0 \\
0 \\
n \\
n\end{array}$ & 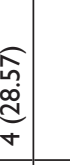 & 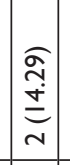 & $\frac{\widehat{c}}{\bar{c}}$ & \\
\hline 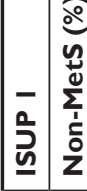 & & 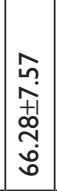 & 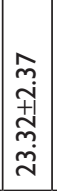 & 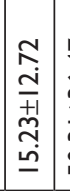 & & $\stackrel{\widehat{N}}{\tilde{n}}$ & 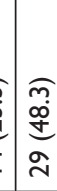 & 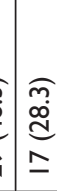 & 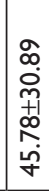 & & 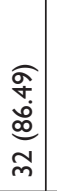 & 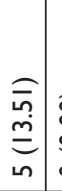 & $\begin{array}{l}\widehat{o} \\
\dot{d} \\
\dot{e}\end{array}$ & & 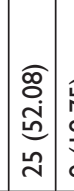 & 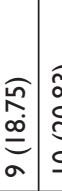 & 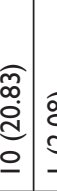 & 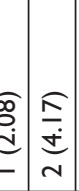 & $\mid \begin{array}{c}\widehat{\alpha} \\
\stackrel{d}{d} \\
-\end{array}$ & 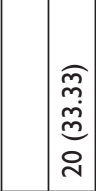 & 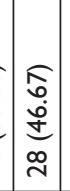 & 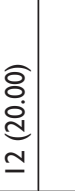 & $\mid \begin{array}{c}\widehat{\widetilde{m}} \\
\infty \\
\stackrel{\infty}{=} \\
=\end{array}$ & 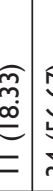 & 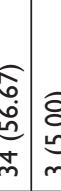 \\
\hline 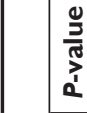 & & 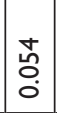 & \begin{tabular}{|l|} 
\\
$\dot{o}$ \\
$\dot{b}$ \\
\end{tabular} & $\frac{0}{0}$ & 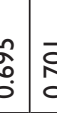 & & & & $\begin{array}{c}\overline{\bar{\infty}} \\
\stackrel{\infty}{\circ}\end{array}$ & $\mid \begin{array}{l}0 \\
0 \\
0 \\
0\end{array}$ & & & & 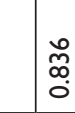 & & & & & & $\mid \begin{array}{l}0 \\
0 \\
0 \\
0 \\
0\end{array}$ & & 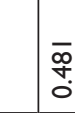 & & & \\
\hline ð̊ & & \begin{tabular}{|l} 
\\
0 \\
0 \\
1 \\
0 \\
0 \\
0 \\
0
\end{tabular} & 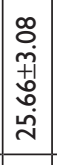 & 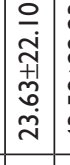 & & & 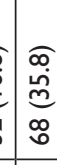 & 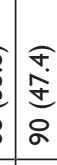 & 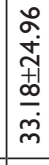 & & 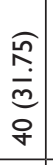 & 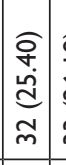 & 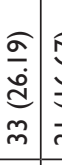 & & 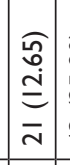 & 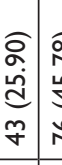 & 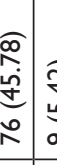 & 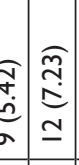 & 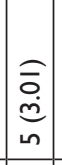 & 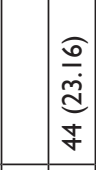 & 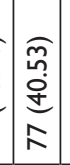 & 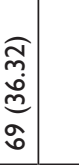 & 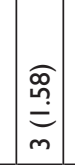 & 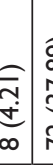 & 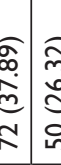 \\
\hline 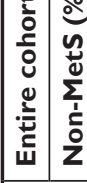 & & 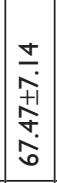 & 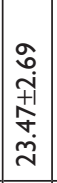 & \begin{tabular}{|l|}
0 \\
0 \\
0 \\
+1 \\
$N$ \\
$\infty$ \\
0 \\
\end{tabular} & & 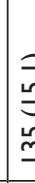 & 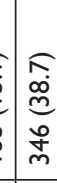 & 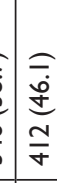 & 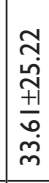 & & 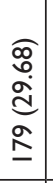 & $\mid \begin{array}{l}\widehat{a} \\
0 \\
a \\
\sigma \\
\underline{\underline{\alpha}} \\
\end{array}$ & 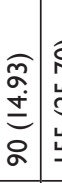 & & $\mid \begin{array}{l}\widehat{\sigma} \\
\hat{\sigma} \\
\hat{\sigma} \\
\underline{\underline{\sigma}}\end{array}$ & 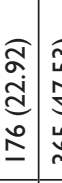 & & 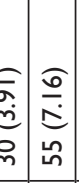 & $\mid \begin{array}{l}\sigma \\
\underset{j}{d} \\
\tilde{\sim}\end{array}$ & 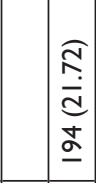 & 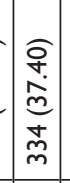 & 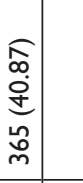 & 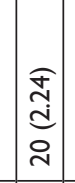 & 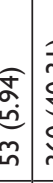 & 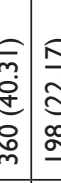 \\
\hline 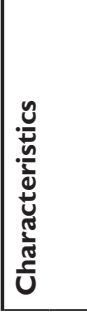 & 号 & 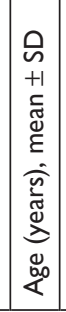 & 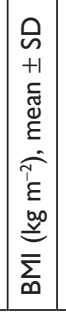 & 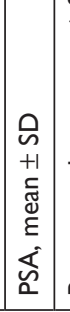 & 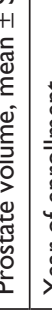 & & 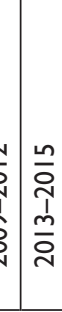 & 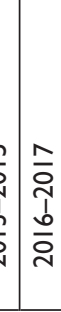 & 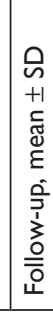 & 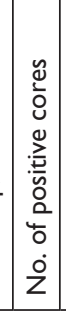 & -1 & 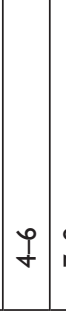 & 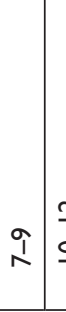 & 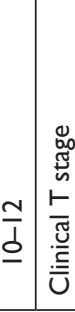 & $\widetilde{F}$ & & & & $\vec{F}$ & 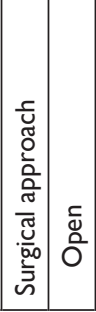 & 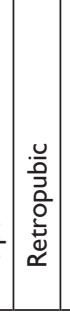 & 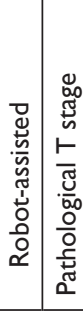 & 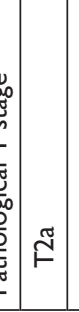 & $\overrightarrow{\tilde{F}}$ & \\
\hline
\end{tabular}




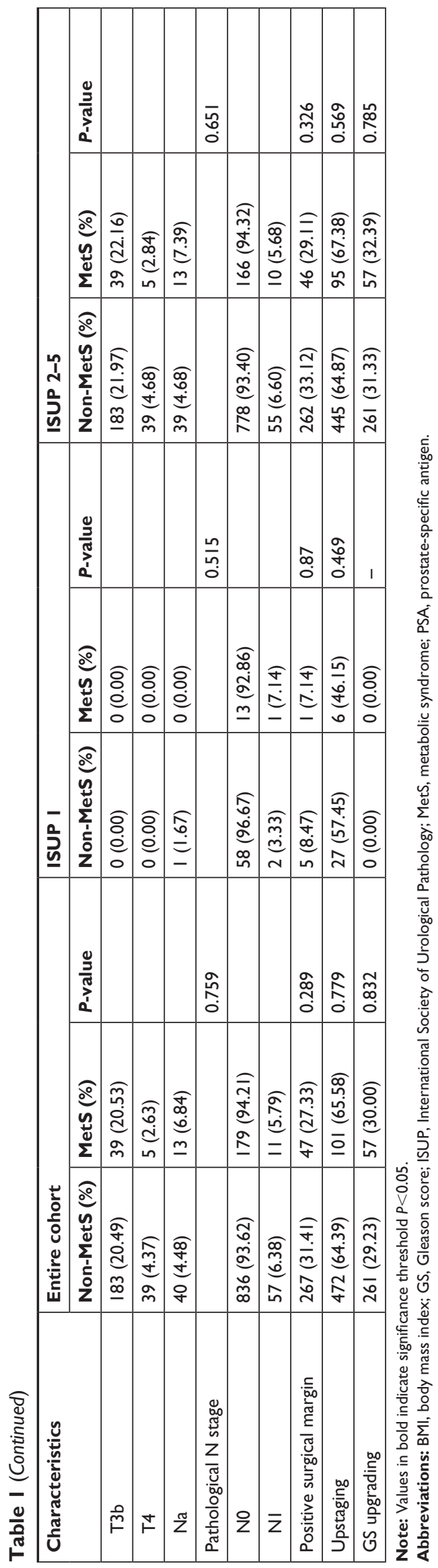

the entire cohort, MetS patients generally had a lower BCRfree survival than non-MetS patients. Eventually, MetS and non-MetS patients had extremely close BCR-free survival, and the difference was not statistically significant. We did not conduct the same analysis for OS rate considering that only 27 deaths occurred. Adjusted Cox hazard regression model was utilized to evaluate the $\mathrm{OS}$ and BCR with a median follow-up time of 40.45 months (3-110 months). The HRs for BCR and death were 0.99 (95\% CI 0.63-1.58, $P=0.9755$ ) and 1.95 (95\% CI 0.44-8.54, $P=0.3761)$.

In the multivariate analysis for number of MetS components (Table 3), an increasing number of MetS risk factors were connected with a greater chance of PSM in the entire cohort ( $P$ for trend $=0.0194)$ and ISUP $2-5$ group $(P$ for trend $=0.0127$ ). In terms of ISUP 1 group, patients with any number of MetS components were associated with a higher probability of PSM than those without any MetS components. In addition, there was a decreasing gradient of PSM probability from one MetS component to three MetS components, although not statistically significant $(P$ for trend $=0.2324$ ).

\section{Discussion}

\section{Key findings and implications}

In the current study, we performed a comprehensive analysis for patients with PCa. Patients were subsequently divided into subgroups based on ISUP grade (ISUP 1 and ISUP 2-5 subgroups) according to the previously reported study, which explored the association between MetS and PCa with GS $=6$ or $\geq 7 .{ }^{18,19}$ Furthermore, we found MetS patients had a higher BMI than non-MetS patients in all the three groups, which is actually unsurprising since BMI is a risk factor of MetS. Prior to our study, Cobelli proved that BMI was an independent indicator of upstaging, upgrading, and seminal vesical invasion. ${ }^{13}$ Additionally, MetS was also reported to be associated with a higher probability of death, upgrading, and PSM. ${ }^{14,20,21}$ Nevertheless, we failed to find any statistical significance with regard to these parameters, even though our findings also indicated MetS in low-risk PCa patients could lead to greater chance of PSM, which is concord with Colicchia et al's conclusion. ${ }^{14}$ Last but not the least, a trend of increasing risk of PSM was observed as the number of MetS components ramped up in the entire cohort and ISUP 2-5 group, and the $P$-value for trend was statistically significant. This is in line with a prior multicenter study which reported a resembling association between PSM and the number of MetS components, except their data showed that having one component would lead to a $25 \%$ decrease of odds ratio than having no component. ${ }^{22}$ To investigate the trend between 
Table 2 Adjusted OR/HR and $95 \% \mathrm{Cl}$ for the pathological outcomes of metabolic syndrome, BCR, and death

\begin{tabular}{|l|l|l|l|l|l|l|}
\hline & Entire cohort & $P$-value & ISUP I & P-value & ISUP 2-5 & $P$-value \\
\hline Upstaging, OR (95\% Cl) & $1.16(0.65,2.07)$ & 0.6245 & $I .55(0.30,7.99)$ & 0.5983 & $1.22(0.64,2.33)$ & 0.5416 \\
\hline Upgrading, OR (95\% Cl) & $1.04(0.73,1.47)$ & 0.8456 & $0.88(0.40,1.94)$ & 0.7475 & $1.10(0.72,1.66)$ & 0.6674 \\
\hline Positive surgical margin, OR (95\% Cl) & $0.97(0.63,1.49)$ & 0.8948 & $1.07(0.32,3.63)$ & 0.9126 & $0.96(0.60,1.52)$ & 0.8588 \\
\hline BCR, HR (95\% Cl) & $0.99(0.63,1.58)$ & 0.9775 & $/$ & $/$ & $1.11(0.68,1.79)$ & 0.6785 \\
\hline Death, HR (95\% Cl) & $1.95(0.44,8.54)$ & 0.3761 & $/$ & $/$ & $3.56(0.77,16.47)$ & 0.1036 \\
\hline
\end{tabular}

Notes: "/” = a small sample size to perform this analysis. Adjusted for BMI, age, PSA, clinical stage, pathological P stage, pathological N stage, surgical approach, and number of positive cores.

Abbreviations: BCR, biochemical recurrence; BMI, body mass index; ISUP, International Society of Urological Pathology; PSA, prostate-specific antigen.
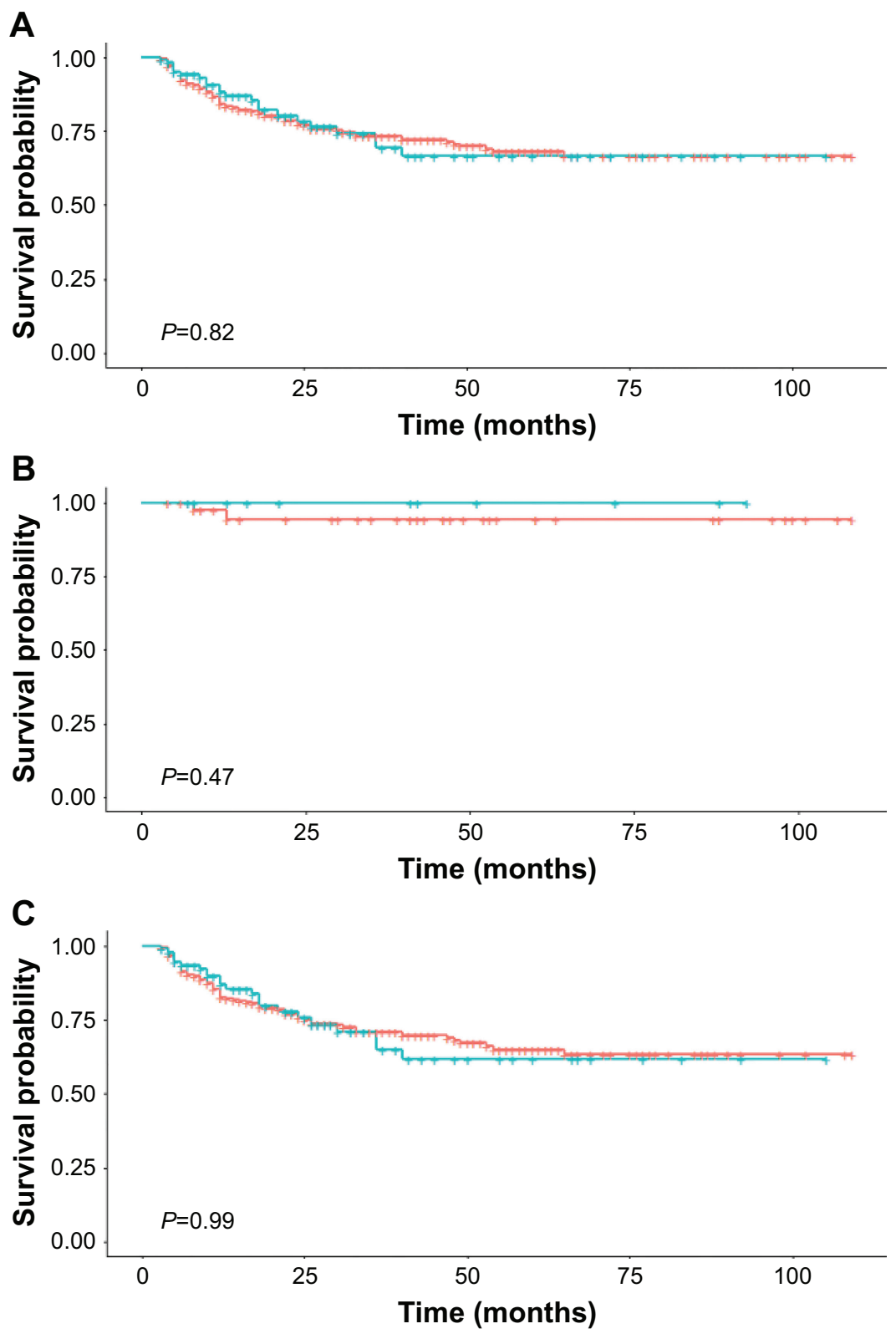

$$
\text { MetS Non-MetS }
$$

Figure I Kaplan-Meier curve for BCR-free survival by metabolic syndrome in the (A) entire cohort, (B) ISUP I group, and (C) ISUP 2-5 group. Abbreviations: BCR, biochemical recurrence; ISUP, International Society of Urological Pathology; MetS, metabolic syndrome. 
Table 3 Adjusted OR and $95 \% \mathrm{Cl}$ for number of metabolic syndrome components for pathological outcomes

\begin{tabular}{|c|c|c|c|}
\hline Pathologic Outcomes & Entire cohort & ISUP I & ISUP 2-5 \\
\hline \multicolumn{4}{|l|}{ Upstaging, OR (95\% Cl) } \\
\hline 0 components & 1 & I & 1 \\
\hline I component & $0.80(0.43,1.45)$ & $0.77(0.27,2.18)$ & $0.98(0.62, I .54)$ \\
\hline 2 components & $0.67(0.36,1.25)$ & $0.80(0.27,2.36)$ & $0.76(0.48,1.22)$ \\
\hline$\geq 3$ components & $0.81(0.38,1.70)$ & I.4I $(0.33,6.09)$ & $0.74(0.44,1.24)$ \\
\hline$P$ for trend & 0.5194 & I & 0.7540 \\
\hline \multicolumn{4}{|l|}{ Upgrading, OR $(95 \% \mathrm{Cl})$} \\
\hline 0 components & 1 & I & 1 \\
\hline I component & $1.23(0.84,1.82)$ & $0.82(0.34,2.02)$ & $1.37(0.85,2.19)$ \\
\hline 2 components & $0.98(0.65,1.47)$ & $0.83(0.32,2.1 \mathrm{I})$ & $1.00(0.60,1.65)$ \\
\hline$\geq 3$ components & $1.10(0.69,1.73)$ & $0.67(0.21,2.14)$ & $1.38(0.81,2.36)$ \\
\hline$P$ for trend & 0.7717 & I & 0.5697 \\
\hline \multicolumn{4}{|c|}{ Positive surgical margin, OR $(95 \% \mathrm{Cl})$} \\
\hline 0 components & 1 & 1 & 1 \\
\hline I component & $1.46(0.8 \mathrm{I}, 2.63)$ & $3.92(0.27,57.06)$ & $1.40(0.75,2.60)$ \\
\hline 2 components & $1.89(1.04,3.46)$ & $3.32(0.18,62.74)$ & $1.85(0.98,3.48)$ \\
\hline$\geq 3$ components & $2.07(1.04,4.15)$ & $2.99(0.09,101.76)$ & $2.05(0.99,4.23)$ \\
\hline$P$ for trend & 0.0194 & 0.2324 & 0.0127 \\
\hline
\end{tabular}

Notes: "l" = a small sample size to perform this analysis. Adjusted for body mass index (BMI), age, prostate-specific antigen (PSA), clinical stage, pathological P stage, pathological $\mathrm{N}$ stage, surgical approach, and number of positive cores. Values in bold indicate significance threshold $P<0.05$.

Abbreviations: BMI, body mass index; ISUP, International Society of Urological Pathology; PSA, prostate-specific antigen.

PSM risk and accumulation of MetS components, we utilized the methodology reported by Park and Lee, ${ }^{23,24}$ who found the same trends between the consumption of coffee with cause-specific mortality and physical activity with weight gain prevention. It implied the necessity to keep a healthy lifestyle and controlling MetS risk factors might reduce the chance of PSM. With regard to the ISUP 1 group, the risk of PSM interestingly declined as the number of MetS components increased from 1 to 3 , but the outcome failed to reach statistical significance. More efforts and studies are required to confirm the trend in ISUP 1 group.

Regarding the increasing risk of PSM with the increase in the number of MetS single components, the possible explanation is that patients diagnosed with MetS and/or its single components (eg, hypertriglyceridemia or hyperglycemia) have a higher risk of extracapsular extension and/or T3-4 pathological stage and/or lymph node involvement, which may subsequently lead to a higher rate of occurrence of PSM among these patients. First, Kheterpal et al found that patients diagnosed with MetS had significantly higher percentage of extracapsular extension ( $34 \%$ vs $26 \%, P=0.003$ ) than those without MetS. ${ }^{25}$ Second, with similarity, Zhang et al found patients diagnosed MetS had significantly higher proportion of T3-4 pathological stage $(48.3 \%$ vs $34.1 \%$, $P<0.001)$ and lymph node involvement $(14.6 \%$ vs $8 \%$,
$P=0.006)$ than patients without MetS. ${ }^{26}$ Moreover, MetS is the independent risk factor of T3-4 pathological stage (OR 1.583, 95\% CI 1.106-2.266, $P=0.012$ ) and lymph node involvement (OR 1.751, 95\% CI 1.038-2.955, $P=0.036$ ). Third and most importantly, Zhang et al also claimed that patients diagnosed with diabetes, hypertriglyceridemia, or low HDL-C had higher proportion of T3-4 pathological stage and lymph node involvement. In detail, their findings are as follows: for patients diagnosed with diabetes: pT3-4 vs pT2 $=21.8 \%$ vs $12.1 \%, P<0.001$; lymph node involvement: Yes vs No $=24.7 \%$ vs $14.7 \%, P=0.011$; for patients diagnosed with hypertriglyceridemia: pT3-4 vs pT2 $=28.8 \%$ vs $22.7 \%$, $P=0.031$; lymph node involvement: Yes vs No $=61.3 \%$ vs $21.2 \%, P<0.001$; for patients confirmed with low HDL-C: pT3-4 vs pT $2=14.5 \%$ vs $10.1 \%, P=0.035$. Last but not the least, in the study of Zhang et al, an ascending trend of the risk of lymph node involvement appeared as the number of MetS components accumulated. ${ }^{26}$ Taken together, our results seem reasonable.

\section{Strengths and limitations}

The present study has several strengths and innovations. First, previous studies were mainly focused on the association between MetS and general any-risk PCa patients, ${ }^{20,21}$ and few studies addressed low-risk patients. ${ }^{14}$ Our study 
performed comprehensive analysis of patients at any risk, who were further divided into low-risk and intermediatehigh risk subgroup. In this case, we were able to have a general understanding of the impact of MetS on patients at different risks. Second, we examined not only MetS, but also the number of MetS components among patients at any risk, which allowed us to explore MetS more deeply. In addition, we employed trend test to confirm our positive findings. Third, we used the most appropriate definition of obesity for Chinese population to include MetS patients in the present study. There are multiple definitions for MetS worldwide, and several Chinese population-based cohorts took BMI over $30 \mathrm{~kg} \mathrm{~m}^{-2}$ as cut-off value for obesity. However, in this study, we utilized the mostly practiced definition of MetS from the American Heart Association (AHA) and the National Heart, Lung, and Blood Institute (NHLBI), which suggests the definition of obesity should be country-specific. Consequently, the definition of obesity from Working Group on Obesity in China, which declared $28 \mathrm{~kg} \mathrm{~m}^{-2}$ as the optimal cut-off point of BMI to define obesity among Chinese population, was employed.

Our findings should be interpreted with following limitations. Retrospective design is the primary limitation, and consequent potential selection bias is a bothering concern for this study. The second limitation is we used BMI rather than waist circumstance to define obesity, which could cause misclassification. Basically, this is a common limitation for urological research. However, as Bhindi et al mentioned in their study, the major discrepancy between these two anthropometric measures occurs in men when they have elevated muscle mass, resulting in elevated BMI but low waist circumference. ${ }^{27}$ This would be an unlikely occurrence given our cohort's age distribution. The last limitation is the number of oncological outcomes (death and BCR) was poor so we could not perform deeper analysis. Given that the 5-year survival rate of localized PCa is approaching 100\%, our follow-up duration is another limitation.

It is noted that surgical approach is one of our concerns that may affect the analytical outcomes even though we adjusted it in multivariate analysis. A recent study by Cochetti demonstrated that nerve sparing technique in RP could lead to increased risk of PSM. ${ }^{28}$ However, our institution began RP with nerve sparing from 2018 and all the patients included in the current cohort were operated without nerve sparing. Hence, future study should probably include this parameter in their analysis and further investigate the impact of nerve sparing technique on oncological and pathological outcomes among MetS patients.

\section{Take home message}

Our study revealed that the accumulation of metabolic syndrome components could lead to a significantly increasing gradient of positive surgical margin risk on the entire $\mathrm{PCa}$ cohort and patients with intermediate-high risk $\mathrm{PCa}$ after prostatectomy but not for the low-risk patients.

\section{Conclusion}

In this study, we performed analysis on PCa patients undergoing RP at any risk, low risk, and intermediate-high risk. We found the accumulation of a number of MetS components can lead to an increasing gradient of PSM risk on the entire cohort and patients with intermediate-high risk (ISUP 2-5). No association was found between MetS and the overall survival, upstaging, upgrading, or BCR.

\section{Acknowledgments}

The authors thank Ian Charles Tobias for reviewing the manuscript. This work was supported by the National Key Research and Development Program of China (Grant No. SQ2017YFSF090096), the Prostate Cancer Foundation Young Investigator Award 2013, the National Natural Science Foundation of China (Grant Nos 81300627, 81370855, 81702536, and 81770756), Programs from Science and Technology Department of Sichuan Province (Grant Nos 2014JY0219 and 2017HH0063), and Young Investigator Award of Sichuan University 2017.

\section{Disclosure}

The authors report no conflicts of interest in this work.

\section{References}

1. Ferlay J, Soerjomataram I, Dikshit R, et al. Cancer incidence and mortality worldwide: sources, methods and major patterns in GLOBOCAN 2012. Int J Cancer. 2015;136(5):E359-E386.

2. Siegel RL, Miller KD, Jemal A. Cancer statistics, 2017. CA Cancer J Clin. 2017;67(1):7-30.

3. Litwin MS, Tan HJ. The diagnosis and treatment of prostate cancer. JAMA. 2017;317(24):2532-2542.

4. Wilt TJ, Brawer MK, Jones KM, et al. Radical prostatectomy versus observation for localized prostate cancer. N Engl J Med. 2012;367(3): 203-213.

5. Bill-Axelson A, Holmberg L, Garmo H, et al. Radical prostatectomy or watchful waiting in early prostate cancer. $N$ Engl J Med. 2014;370(10): 932-942.

6. Alberti KG, Eckel RH, Grundy SM, et al. Harmonizing the metabolic syndrome: a joint interim statement of the International diabetes Federation Task Force on epidemiology and prevention; National Heart, lung, and Blood Institute; American Heart Association; World Heart Federation; International Atherosclerosis Society; and International Association for the Study of Obesity. Circulation. 2009;120(16):1640-1645.

7. Jaggers JR, Sui X, Hooker SP, et al. Metabolic syndrome and risk of cancer mortality in men. Eur J Cancer. 2009;45(10):1831-1838. 
8. Hursting SD, Hursting MJ. Growth signals, inflammation, and vascular perturbations: Mechanistic links between obesity, metabolic syndrome, and cancer. Arterioscler Thromb Vasc Biol. 2012;32(8):1766-1770.

9. Braun S, Bitton-Worms K, Leroith D. The link between the metabolic syndrome and cancer. Int J Biol Sci. 2011;7(7):1003-1015.

10. De Nunzio C, Aronson W, Freedland SJ, Giovannucci E, Parsons JK. The correlation between metabolic syndrome and prostatic diseases. Eur Urol. 2012;61(3):560-570.

11. Harding J, Sooriyakumaran M, Anstey KJ, et al. The metabolic syndrome and cancer: is the metabolic syndrome useful for predicting cancer risk above and beyond its individual components? Diabetes Metab. 2015;41(6):463-469.

12. Bhindi B, Kulkarni GS, Finelli A, et al. Obesity is associated with risk of progression for low-risk prostate cancers managed expectantly. Eur Urol. 2014;66(5):841-848.

13. de Cobelli O, Terracciano D, Tagliabue E, et al. Body mass index was associated with upstaging and upgrading in patients with low-risk prostate cancer who met the inclusion criteria for active surveillance. Urol Oncol. 2015;33(5):201.e1-201.e8.

14. Colicchia M, Morlacco A, Rangel LJ, Carlson RE, Dal Moro F, Karnes RJ. Role of metabolic syndrome on perioperative and oncological outcomes at radical prostatectomy in a low-risk prostate cancer cohort potentially eligible for active surveillance. Eur Urol Focus. Epub 2018 Jan 3.

15. Lund Håheim L, Wisløff TF, Holme I, Nafstad P. Metabolic syndrome predicts prostate cancer in a cohort of middle-aged Norwegian men followed for 27 years. Am J Epidemiol. 2006;164(8):769-774.

16. Ford ES, Mokdad AH, Giles WH. Trends in waist circumference among U.S. adults. Obes Res. 2003;11(10):1223-1231.

17. Zhou BF, Cooperative Meta-Analysis Group of the Working Group on Obesity in China. Predictive values of body mass index and waist circumference for risk factors of certain related diseases in Chinese adults - study on optimal cut-off points of body mass index and waist circumference in Chinese adults. Biomed Environ Sci. 2002;15(1): 83-96.

18. De Nunzio C, Simone G, Brassetti A, et al. Metabolic syndrome is associated with advanced prostate cancer in patients treated with radical retropubic prostatectomy: results from a multicentre prospective study. BMC Cancer. 2016;16(1):407.
19. De Nunzio C, Freedland SJ, Miano R, et al. Metabolic syndrome is associated with high grade Gleason score when prostate cancer is diagnosed on biopsy. The Prostate. 2011;71(14):1492-1498.

20. Léon P, Seisen T, Cussenot O, et al. Low circulating free and bioavailable testosterone levels as predictors of high-grade tumors in patients undergoing radical prostatectomy for localized prostate cancer. Urol Oncol. 2015;33(9):384.e21-e27.

21. Shiota M, Yokomizo A, Takeuchi A, et al. The feature of metabolic syndrome is a risk factor for biochemical recurrence after radical prostatectomy. J Surg Oncol. 2014;110(4):476-481.

22. Lebdai S, Mathieu R, Leger J, et al. Metabolic syndrome and low highdensity lipoprotein cholesterol are associated with adverse pathological features in patients with prostate cancer treated by radical prostatectomy. Urol Oncol. 2018;36(2):80.e17-e17.

23. Park SY, Freedman ND, Haiman CA, et al. Association of coffee consumption with total and cause-specific mortality among Nonwhite populations. Ann Intern Med. 2017;167(4):228-235.

24. Lee IM, Djousse L, Sesso HD, Wang L, Buring JE. Physical activity and weight gain prevention. JAMA. 2010;303(12):1173-1179.

25. Kheterpal E, Sammon JD, Diaz M, et al. Effect of metabolic syndrome on pathologic features of prostate cancer. Urol Oncol. 2013;31(7): 1054-1059.

26. Zhang GM, Zhu Y, Dong DH, et al. The association between metabolic syndrome and advanced prostate cancer in Chinese patients receiving radical prostatectomy. Asian J Androl. 2015;17(5):839-844.

27. Bhindi B, Locke J, Alibhai SMH, et al. Dissecting the association between metabolic syndrome and prostate cancer risk: analysis of a large clinical cohort. Eur Urol. 2015;67(1):64-70.

28. Cochetti G, Boni A, Barillaro F, et al. Full neurovascular sparing extraperitoneal robotic radical prostatectomy: our experience with PERUSIA technique. J Endourol. 2017;31(1):32-37.
OncoTargets and Therapy

\section{Publish your work in this journal}

OncoTargets and Therapy is an international, peer-reviewed, open access journal focusing on the pathological basis of all cancers, potential targets for therapy and treatment protocols employed to improve the management of cancer patients. The journal also focuses on the impact of management programs and new therapeutic agents and protocols on

\section{Dovepress}

patient perspectives such as quality of life, adherence and satisfaction The manuscript management system is completely online and includes a very quick and fair peer-review system, which is all easy to use. Visit http://www.dovepress.com/testimonials.php to read real quotes from published authors. 